OPEN ACCESS

Edited by:

Giovanni Di Pino,

Campus Bio-Medico University, Italy

Reviewed by:

Nils Henrik Pixa,

University of Münster, Germany

Tomofumi Yamaguchi,

Juntendo University, Japan

Daniel Gomes Da Silva Machado,

Federal University of Rio Grande do

Norte, Brazil

Elisabeth Kaminski,

Leipzig University, Germany

*Correspondence:

Sophie C. Andrews

s.andrews@neura.edu.au

Specialty section:

This article was submitted to

Motor Neuroscience,

a section of the journal

Frontiers in Human Neuroscience

Received: 17 February 2021 Accepted: 21 May 2021

Published: 16 June 2021

Citation:

Quinlan C, Rattray B, Pryor D, Northey JM, Coxon J, Cherbuin N and Andrews SC (2021) A Short-Term Intervention of High-Intensity Exercise and Anodal-tDCS on Motor Learning

in Middle-Aged Adults: An RCT.

Front. Hum. Neurosci. 15:661079.

doi: 10.3389/fnhum.2021.661079

\section{A Short-Term Intervention of High-Intensity Exercise and Anodal-tDCS on Motor Learning in Middle-Aged Adults: An RCT}

\author{
Clare Quinlan 1,2, Ben Rattray',2,3, Disa Pryor², Joseph M. Northey ${ }^{1,2,3}$, James Coxon ${ }^{4}$, \\ Nicolas Cherbuin ${ }^{3}$ and Sophie C. Andrews ${ }^{3,4,5,6 *}$
}

${ }^{1}$ UC Research Institute for Sport and Exercise, University of Canberra, Canberra, ACT, Australia, ${ }^{2}$ Discipline of Sport and Exercise Science, Faculty of Health, University of Canberra, Canberra, ACT, Australia, ${ }^{3}$ Centre for Research on Ageing, Health and Wellbeing, Australian National University, Canberra, ACT, Australia, ${ }^{4}$ Turner Institute for Brain and Mental Health, School of Psychological Sciences, Monash University, Clayton, VIC, Australia, ${ }^{5}$ Neuroscience Research Australia, Randwick, NSW, Australia, ${ }^{6}$ School of Psychology, University of New South Wales, Sydney, NSW, Australia

High-intensity exercise has enhanced motor learning in healthy young adults. Anodaltranscranial direct current stimulation (a-tDCS) may optimize these effects. This study aimed to determine the effects of a short-term high-intensity interval exercise intervention either with or without a-tDCS on the learning and retention of a novel motor task in middle-aged adults. Forty-two healthy middle-aged adults (age $=44.6 \pm 6.3$, female $=76 \%$ ) were randomized into three groups: exercise and active a-tDCS, exercise and sham a-tDCS, and a non-exercise and sham a-tDCS control. Participants completed a baseline testing session, followed by three intervention sessions 48-h apart. The exercise groups completed 20-min of high-intensity exercise followed by a novel sequential visual isometric pinch task (SVIPT) while receiving 20-min of $1.5 \mathrm{~mA}$ a-tDCS, or sham tDCS. The control group completed 20-min of reading before receiving sham a-tDCS during the SVIPT. Learning was assessed by skill change within and between intervention sessions. Participants returned 5-7 days after the final intervention session and performed the SVIPT task to assess retention. All three groups showed evidence of learning on the SVIPT task. Neither group displayed enhanced overall learning or retention when compared to the control group. High-intensity exercise with or without a-tDCS did not improve learning or retention of a novel motor task in middle-aged adults. The methodological framework provides direction for future research to investigate the potential of differing exercise intensity effects on learning and retention.

Keywords: cognition, non-invasive brain stimulation, motor cortex, aerobic exercise, transcranial direct current stimulation

\section{INTRODUCTION}

Aging is associated with declines in motor function and control which can cause increased cognitive effort in functional tasks (Seidler et al., 2010), as well as difficulty maintaining employability and safety in the aging workforce (Kowalski-Trakofler et al., 2005). Thus, the development of strategies which optimize the acquisition and retention of motor-skills are crucial in maintaining motor 
function and enabling healthy aging. Middle adulthood is a critical window for interventions targeted at delaying the onset of symptom-related cognitive decline (Macpherson et al., 2017), and may be an appropriate time to target motor-skill learning.

Participating in exercise can have positive benefits on brain function, dependent on the type of exercise, as well as the cognitive domain targeted (Mandolesi et al., 2018). Compared to low intensity exercise, which refers to exercise at $28-39 \%$ of $\mathrm{VO}_{2 \max }$, or $45-54 \%$ of a person's maximum heart rate (\%HRmax), high intensity exercise can range from $60 \%$ of $\mathrm{VO}_{2 \max }$ and $70 \% \mathrm{HRmax}$ to $100 \%$, representing a maximal effort (Vanhees et al., 2012; Buchheit and Laursen, 2013). High-intensity interval exercise consists of repeated efforts of exercise ranging from less than $45 \mathrm{~s}$ to $2-4 \mathrm{~min}$ efforts (Buchheit and Laursen, 2013), For motor learning, a single session of high-intensity-interval exercise can benefit learning between sessions, when performed $20 \mathrm{~min}$ after learning, more than lower intensity exercise (Thomas et al., 2016). Exercise performed approximately $20 \mathrm{~min}$ before learning has also been observed to increase motor skill learning (Mang et al., 2014), and consolidation (Stavrinos and Coxon, 2017). High-intensity exercise performed immediately prior to task practice (Skriver et al., 2014), and following task practice (Roig et al., 2012) resulted in higher retention at 7 days compared to non-exercise control groups. However, this has not yet been investigated in middle-aged adults (35-55 years). High-intensity exercise may contribute to a cascade of neuromodulators beneficial to increased neuronal plasticity and long-term potentiation (Loprinzi et al., 2019). This may include a transient increase in brain-derived neurotrophic factor (BDNF) which appears to display a dose-response relationship with exercise intensity, in which high-intensity exercise increases BDNF above that of continuous exercise (Saucedo Marquez et al., 2015). Further, high-intensity exercise can increase lactate, which crosses the blood-brain barrier, and can enhance synaptic plasticity (Yang et al., 2014). High-intensity exercise has also been shown to increase the neuroplastic response to intermittent theta burst stimulation more so than moderate-intensity exercise in healthy adults (Andrews et al., 2020). Supporting this, a recent meta-analysis of 22 studies investigating the effects of acute cardiovascular exercise on motor learning and memory task performance (Wanner et al., 2020), concluded that high intensity exercise could be beneficial to motor memory consolidation.

Optimization of the benefits of exercise may occur by its combination with other potential neuro-enhancing methods, such as non-invasive brain stimulation. It is posited that transcranial direct current stimulation (tDCS) can be used to alter neuronal excitability. Anodal-tDCS (a-tDCS) is thought to increase cortical stimulation by subthreshold depolarization of neurons in the target region of stimulation. This has been demonstrated in pharmacological investigations showing elimination of excitability effects of a-tDCS when voltage-gated sodium channels are blocked, and a reduction in excitability when calcium channels are blocked (Nitsche et al., 2003a). Although the precise mechanisms are not fully understood (Bestmann et al., 2015), a-tDCS may enhance cognitive function by increasing the chance of neuronal firing. However, there is increasing recognition that these responses may be highly variable due to dose administration, and biological responses to the administered stimuli (Li et al., 2015). On its own, when applied during a motor task, a number of studies have demonstrated that a-tDCS has enhanced the learning and consolidation of a motor task (Nitsche et al., 2003b; Reis et al., 2009), as well as the rate of learning compared to c-tDCS (Stagg et al., 2011). Additionally, a-tDCS may act to optimize the effects of exercise on cognitive training outcomes through shared mechanisms (Ward et al., 2017; Steinberg et al., 2019), and this synergistic benefit may also be seen in the context of motor learning. One such mechanism, may be the shared involvement of BDNF. BDNF has been implicated in long-term potentiation and long-term memory formation (Cunha et al., 2010), exemplified by "rescue protocols," in which treatment with BDNF largely revered the synaptic deficits observed in BDNF knockout mice brain (Pozzo-Miller et al., 1999). As described above, exercise increases BDNF in a dose response manner (Schmolesky et al., 2013; Saucedo Marquez et al., 2015) with high-intensity exercise increasing BDNF more so than moderate intensity exercise (Schmolesky et al., 2013). As BDNF has been shown to be a key modulator in longterm potentiation induced by direct current stimulation (Fritsch et al., 2010), it has been posited these pathways may converge allowing for the increase in BDNF following exercise to form an optimal environment for successful tDCS induced long-term potentiation (Steinberg et al., 2019). In this instance, exercise may act as a primer, rendering the synapses more responsive to future stimulation (Kronberg et al., 2017; Steinberg et al., 2019).

In young adults, combining both a high-intensity cardiovascular and resistance exercise protocol and a-tDCS within the same intervention, resulted in greater improvements on tests of intelligence compared to either intervention alone in a 4-month randomized controlled trial, however, the timing between intervention components was not described (Ward et al., 2017). High-intensity interval exercise may be more effective in enhancing neuroplasticity induced by brain stimulation when performed prior to, instead of after, a brain stimulation protocol (Mellow et al., 2020). To date, the investigators are not aware of any published research investigating the combined effects of exercise and a-tDCS on motor-skill learning. Positive effects of a single session of high-intensity exercise on motor-skill learning and retention (Roig et al., 2012; Mang et al., 2014; Thomas et al., 2016; Stavrinos and Coxon, 2017) have been demonstrated in healthy young adults. However, to date, the effects of high-intensity interval exercise, over multiple days has not been investigated. While it has been hypothesized that the effects of a-tDCS and exercise may be additive to improving cognitive enhancement (Steinberg et al., 2019), this has not been investigated in motor-learning. This study aimed to investigate the hypothesis that a short-term (3 day) high-intensity interval exercise intervention would enhance motor-skill learning and retention over multiple days compared to no exercise in middle-aged adults, and that the benefits would be further enhanced through combination with a-tDCS applied during the completion of the motor learning task. 


\section{MATERIALS AND METHODS}

\section{Participants}

Participants aged 35-55 were recruited through the distribution of flyers, social media, and word of mouth. Included participants had no known history of neurological impairment, no current use of psychoactive medications (e.g., anti-depressants), no contraindications to tDCS (DaSilva et al., 2011; Antal et al., 2017), no diagnosed color blindness, had 20/20 corrected vision, and were safe to undertake high-intensity exercise, as assessed by a pre-exercise screening questionnaire [Adult Pre-Screening Exercise Tool VI (2011), ESSA]. The Goldberg Anxiety and Depression Scale (Goldberg et al., 1988) was used to assess participants' self-reported depression and anxiety symptoms to ensure homogeneity across all groups at baseline. Forty-two participants (age $=44.6 \pm 6.3$, female $=32$, left handed $=3$ ), were included in the final analysis. Participants were considered physical active, on average reporting $512 \pm 675$ min of moderatevigorous physical activity (MVPA) per week.

In consultation with a statistician, and using validated online power-analysis software (Schoenfeld, 2015), an a priori sample size of 42 participants was estimated would provide sufficient power to detect significant differences $(\alpha=0.05 ; \beta=0.80)$ for a three-condition study with repeated measures. This power analysis was calculated using findings from a previous study that has shown an estimated mean difference in skill of $0.19 \pm 0.11$ on the same motor task, with a similar exercise protocol as this study, in young adults (Stavrinos and Coxon, 2017). Based on previous experience, we aimed to recruit a minimum of 51 participants to allow for a $10 \%$ drop out rate, and a $10 \%$ exclusion rate for equipment errors resulting in missing data. Forty-five participants completed the full study protocol, however, three were excluded due to concerns with recording error $(n=2)$ and tDCS application (repeated impedance errors during a sessions) $(n=1)$, As such, 42 participants were included in the analysis (see Figure 1).

The study protocol was approved by the University of Canberra and the Australian National University Human Research Ethics Committee (HREC 16-121, and 2016/322, respectively) and all participants provided written informed consent before commencement.

\section{Study Design}

A three-arm randomized-controlled trial was conducted. On completion of screening and written informed consent, participants were randomized to one of three experimental groups stratified for sex: (1) an exercise group with sham (inactive) tDCS, (2) an exercise group with active a-tDCS, or (3) a no exercise (control) group with sham tDCS. The randomization was completed by a researcher external to the data collection process, utilizing an online random number generator (randomizer.org). Double blinding of the tDCS protocol was applied to all participants, and the researchers involved in data collection until after data were cleaned for analysis. All participants attended the laboratory to complete a standardized baseline session before returning at least 48-h

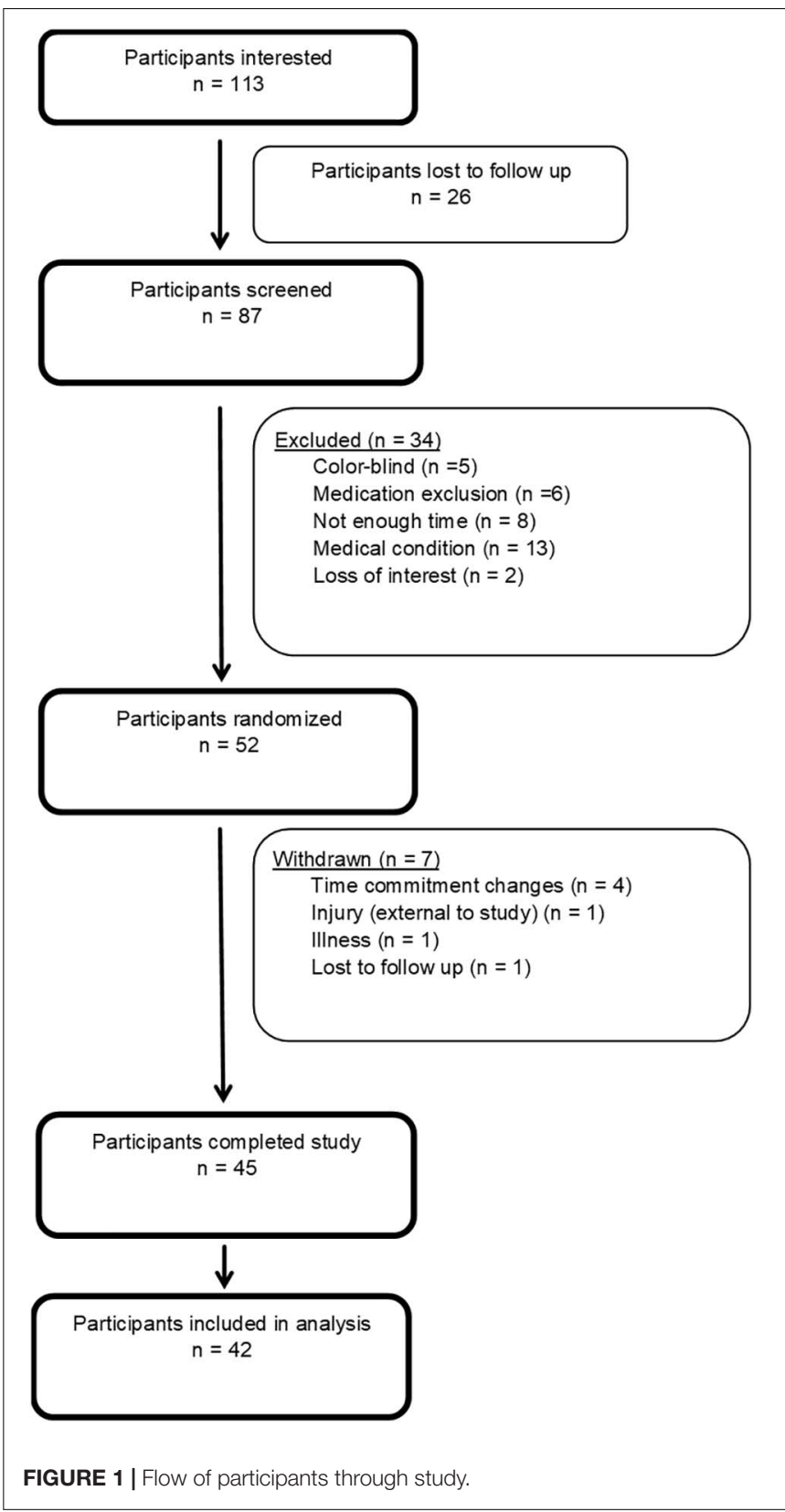

later to complete three intervention sessions over 5 days, with 48-h between sessions (Figure 2). Participants completed a standardized retention session 5-7 days later. Participants were asked to refrain from strenuous exercise, caffeine, and alcohol in the 24-h before all testing sessions.

\section{Procedures}

\section{Baseline Testing}

During baseline testing, participants' height, weight, anxiety and depression symptomatology were recorded. Participants then completed a task of manual dexterity, a short battery of cognitive tasks, and a graded exercise test on a cycle ergometer (HighPerformance Ergometer, Schoberer Rad MeBtechnik, Germany). 


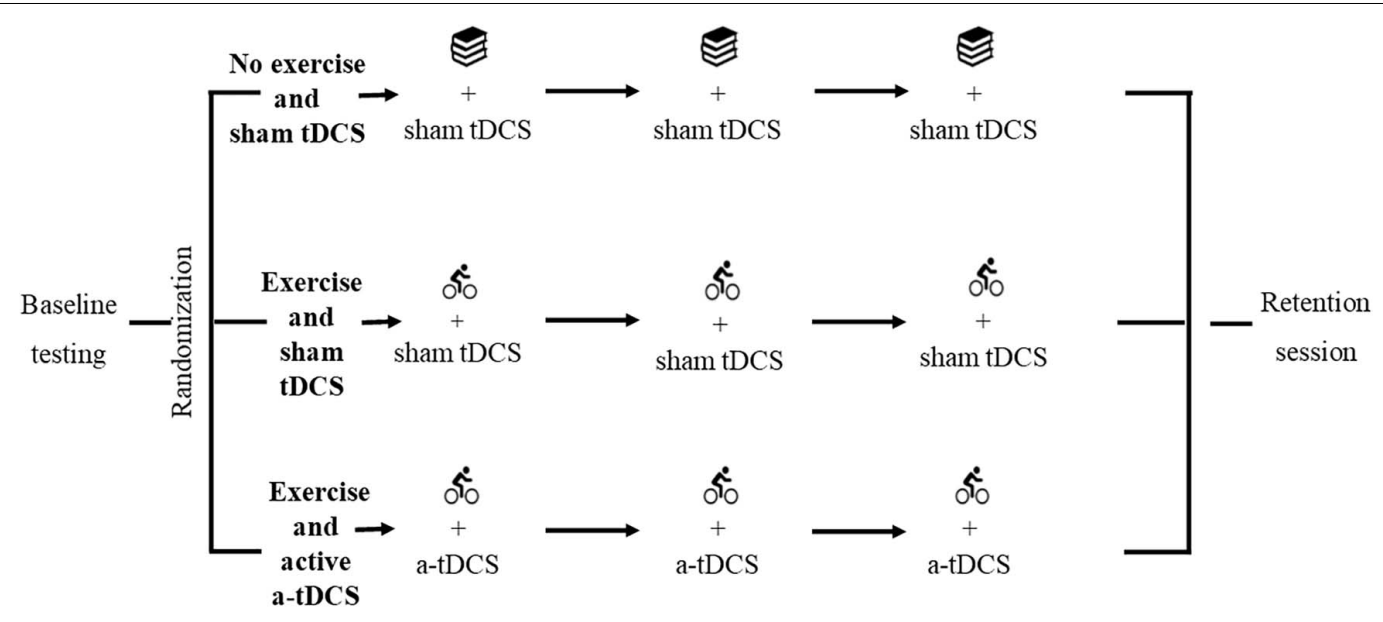

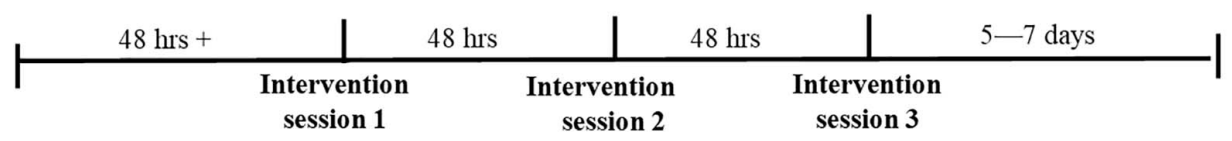

FIGURE 2 | Study design. Participants were randomized following standardized baseline testing. Each group then completed their assigned protocol at each of the three intervention sessions. The exercise and active a-tDCS group performed 20 min of cycling, followed by the learning task with active a-tDCS. The exercise and sham tDCS group performed 20 min of cycling, followed by the learning task with sham tDCS. The non-exercise and sham tDCS group performed 20 min of quiet reading, followed by the learning task with sham tDCS. The learning task and relevant tDCS protocol were applied within 10 min of the completion of exercise. All participants then completed a standardized retention session 5-7 days later.

\section{Intervention Sessions}

\section{Exercise and Active a-tDCS}

Participants exercised for 20-min on a cycle ergometer (HighPerformance Ergometer, Schoberer Rad MeBtechnik, Germany). The protocol shared similarities to previous studies (Roig et al., 2012; Mang et al., 2014; Stavrinos and Coxon, 2017). Participants completed a 4-min warm-up at $50 \mathrm{~W}$, except in two instances where, for participant comfort, due to exceptional fitness 80 $\mathrm{W}$ was used, and one instance of low fitness $20 \mathrm{~W}$ was used, at a self-selected cadence. Participants then completed four 2min epochs of cycling at $90 \%$ of the peak load achieved during the graded exercise test at a cadence of 90 revolutions per minute (RPM) interspersed by 2 -min of active recovery at 50 to $80 \mathrm{~W}$, to accommodate participant comfort, and self-selected cadence. Heart rate (Polar Electro, Kempele, Finland) and rating of perceived exertion (6-20 grade scale, Borg) were monitored before and after each high-intensity epoch.

Within $10 \mathrm{~min}$ following the exercise, participants were fitted with a STARSTIM (Neuroelectrics, Barcelona, Spain) tDCS cap with two saline-soaked $25 \mathrm{~cm}^{2}$ sponge-based electrodes. Participants received 20-min of $1.5 \mathrm{~mA}$ bi-hemispheric a-tDCS with a 30-s ramp-up and ramp-down period. The anodal stimulation applied over the dominant motor cortex (M1) (C3/C4), determined by participant handedness, which has been previously shown to increase acquisition of motor movements, and the cathodal return electrode situated over the nondominant M1 (Waters-Metenier et al., 2014). Treatments were administered in a double-blind manner. During this 20-min, participants completed four blocks of a motor-skill task: the SVIPT.

\section{Exercise and Sham tDCS}

Participants in this group undertook the same exercise protocol as above. The only difference was following the exercise participants received sham-tDCS. The electrical current was only administered for the first and last $30 \mathrm{~s}$.

\section{Non-exercise and Sham tDCS}

Participants in this group completed the same sham tDCS protocol as the Exercise and sham tDCS group above, however, 20-min of quiet reading replaced the exercise.

\section{Retention Assessment}

5-7 days after the final intervention sessions, all participants returned to complete two blocks of the SVIPT task to measure skill retention. The task was preceded with 20 -min of quiet reading to ensure all participants were at a similar level of arousal prior to testing. No exercise, a-tDCS, or sham tDCS was administered during this session. Participants reported which tDCS protocol they thought they received during their intervention sessions.

\section{MEASURES}

\section{Primary Outcome Measure}

The primary outcome measure was motor-skill learning assessed by the performance on a SVIPT (Reis et al., 2009), which was novel to all participants. The implementation of the SVIPT was similar to previous reports (Reis et al., 2009; Stavrinos and Coxon, 2017), utilizing MATLAB software (MATLAB 2016a, The MathWorks, Inc., MA, United States). Participants received 
standardized instructions and familiarization to the SVIPT presented both visually and verbally. Participants were seated at a laptop computer, with a 15-inch screen, and held a force transducer with the thumb and first finger of their dominant hand, determined by self-report, keeping their digits straight. Pinching the force transducer created force pulses which moved the cursor on the screen, from a black (home) tab at the left of the screen, in a horizontal motion to the right. The targets changed from blank to colored to indicate the beginning of the trial, appearing on screen from left to right in the order BLUE - WHITE - GREEN - RED - YELLOW. Participants were instructed to navigate the cursor from the home tab to the five colored targets, displayed in Figure 3, returning to the home tab in between each target in the order RED BLUE - GREEN - YELLOW - WHITE, emphasizing speed and accuracy equally, with each repetition a single trial. A visual reminder of the order was presented above the laptop during all trials. This order was consistent across all trials and participants (Reis et al., 2009). There were eight trials per block, and four blocks per intervention session and two blocks in the retention session. Visual feedback was provided after each block through a graphic representation of the average speed and accuracy of the completed block, and written and verbal feedback comparing the current block to the previous block, which could not be skipped by participants. The target centers were set to $8.75,17.5,26.25$, 35 , and 43.75 percent of the participants maximal voluntary contraction (MVC), respectively. MVC was determined prior to the familiarization trial, which took place on intervention day one, prior to the intervention session, by participants briefly pinching the force transducer, as described above, at their maximal capacity. The highest MVC of three attempts was used. For familiarization, participants completed two blocks of the learning task. Motor-skill was determined by calculating a skill measure, as described previously (Reis et al., 2009; Stavrinos and Coxon, 2017). Task performance of the SVIPT consisted of three components (1) online motor-skill change: assessed by comparing the average motor-skill of each block throughout the learning phase, (2) offline motor-skill change: assessed by assessing the average motor-skill level of the final block of each intervention session to that of the first block of the proceeding session, and (3) retention of motor-skill: assessed by comparing the average motor-skill from the final intervention session, to that of the two retention blocks.

\section{Participant Descriptives Depression and Anxiety Symptomatology}

Symptoms of depression and anxiety may cause disturbances in motor-skill learning (Caligiuri and Ellwanger, 2000). Depression and anxiety symptomatology were assessed using the Goldberg Anxiety and Depression Scale (Goldberg et al., 1988) to allow for group comparison.

\section{Manual Dexterity}

To allow for the comparison of participants manual dexterity at baselines, participants completed the first three stages of the Purdue Pegboard task (Lafayette Instrument Company, 2015). Participants inserted as many pegs into the board within a 30-s period, with their dominant hand, their non-dominant hand, and finally with both hands together.

\section{Cognitive Function}

A battery of computerized cognitive tasks was used to test group similarity at baseline. The battery assessed working memory, attention, processing speed, set-shifting, and inhibitory control as these domains may play an increased role in motor functioning with age (Ren et al., 2013). All tasks were designed and administered using E-Prime 3.0 software (Psychology Software Tools, Pittsburgh, PA, United States).

Working memory was assessed using a Sternberg task, consisting of trials with a stimulus encoding phase and memory retrieval phase (Kober et al., 2016). Selective attention was assessed using a Stroop task (Stroop, 1935), with a working set of 102 trials, with $70 \%$ incongruent trials. A Go/No-Go task employing two task levels of increasing difficulty assessed attention, processing speed, and set-shifting in the first stage, and inhibitory and cognitive control in the latter task level (Langenecker et al., 2007). Cognitive flexibility and set-shifting were assessed using a 64 card Wisconsin Card Sorting task (Fox et al., 2013).

\section{Cardiorespiratory Fitness}

Participants completed a graded exercise test on a cycle ergometer (High-Performance Ergometer, Schoberer Rad MeBtechnik, Germany). Participants completed a 3-min warm-up at a selfselected cadence. The starting protocol commenced at $50 \mathrm{~W}$ for females $(20 \mathrm{~W}, n=1)$ and $100 \mathrm{~W}$ for males $(150 \mathrm{~W}, n=1)$ and was increased every $2 \mathrm{~min}$ by $10-30 \mathrm{~W}$, to ensure participants reached maximal exertion $\sim 12-15$ min after completion of the warmup. Expired gases were collected using Hans-Rudolph facemasks and were analyzed as an average over $20 \mathrm{~s}$ to calculate the peak oxygen uptake $\left(\mathrm{VO}_{2 \text { peak }}\right)$ (Vyntus CPX Metabolic Cart, Jaeger, Germany). Heart rate (Polar Electro, Kempele, Finland) was monitored throughout each trial. The test ceased when participants were unable to maintain a cadence of $>60$ RPM for more than $30 \mathrm{~s}$.

\section{Data Processing}

Prior to data analysis, the SVIPT data were cleaned. Single trials were excluded if there were more than five force pulses and the incorrect order of colors was used, or if pulses were not accurately recorded. The primary outcome measure of motorskill was calculated from the speed and accuracy of the force peaks in each trial. Speed was calculated as the time from the appearance of the colored targets, to the completion of the last pulse force of the trial. Trial force error is a measure of the sum of differences between a target, and the respective force peak of the participants attempt to hit the target over the five trials within a block. Subsequently, the average skill was calculated using the skill parameter calculation (Eq. 1), where a larger value represents better performance (Stavrinos and Coxon, 2017). Although the beta value in the below equation was developed in younger adults, it is similar to those previously utilized in older adults (Mooney et al., 2019), and was used across all participants, therefore not affecting differences in skill. The skill parameter has previously 
A

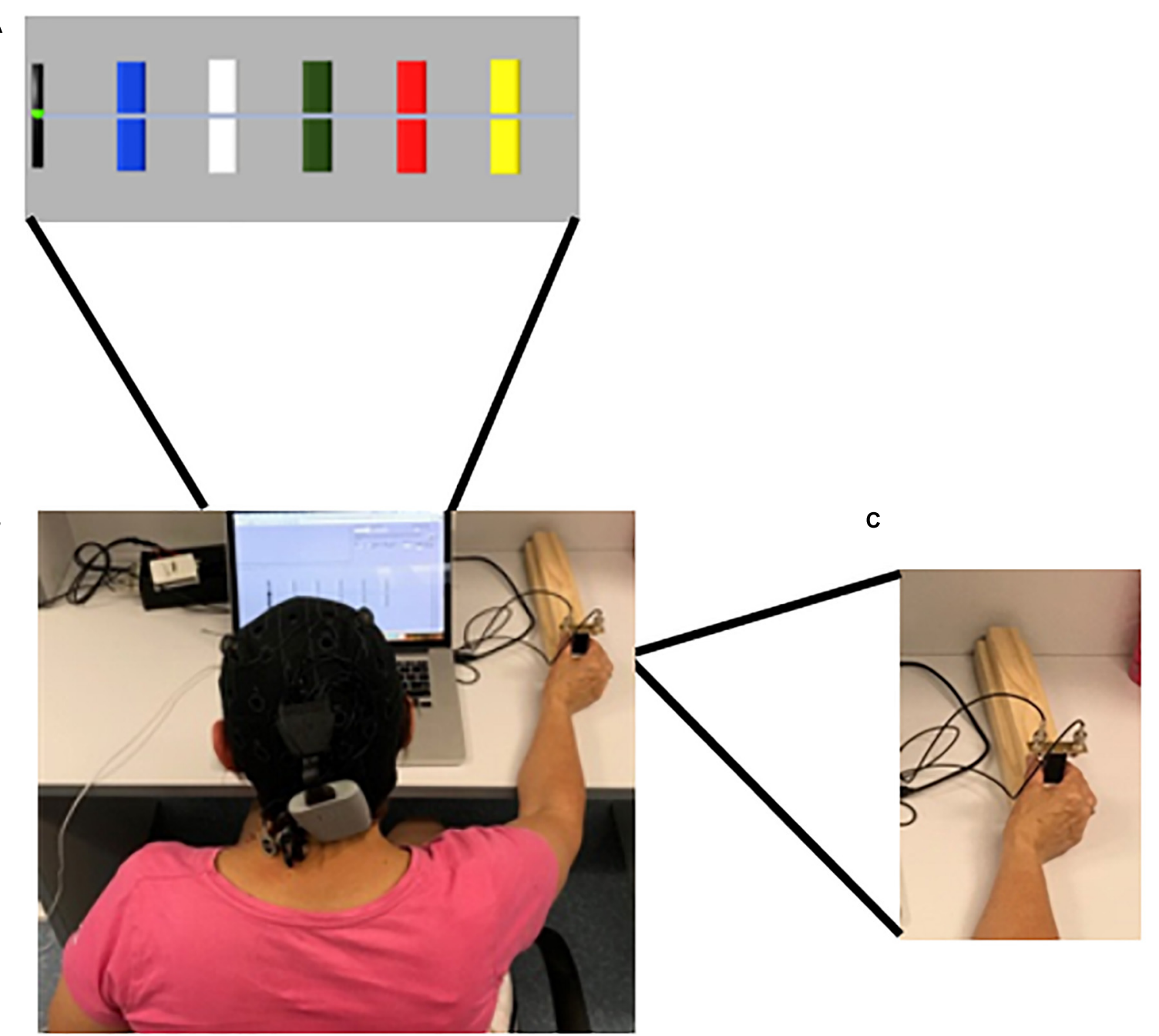

FIGURE 3 | Sequential visual isometric pinch task (SVIPT). (A) Representation of SVIPT task presented on screen to participants. The Colors were presented from left to right in the order BLUE - WHITE - GREEN - RED - YELLOW. Participants were instructed to hit the targets in the order RED - BLUE - GREEN - YELLOW WHITE, returning to the (black) home tab between each. (B) tDCS cap with electrodes. Participants randomized into an active-tDCS group were fitted with a tDCS cap with two saline-soaked $25 \mathrm{~cm}^{2}$ sponge-based electrodes. 20-min of $1.5 \mathrm{~mA}$ bi-hemispheric a-tDCS with the anodal stimulation applied over the dominant motor cortex (M1) (C3/C4), determined by participant handedness. For participants randomized to the sham-tDCS group, a $30 \mathrm{~s}$ ramp period was applied at the beginning and the end of the 20-min period only. (C) The cursor was moved between the home tab to each of the required colors in a horizontal motion by pinching the force transducer between the thumb and the first finger of the dominant hand.

been log-transformed to reduce the heteroscedasticity of the data (Reis et al., 2009; Stavrinos and Coxon, 2017), however, sensitivity analysis revealed that this technique did not alter the results of the current study.

$$
\text { Skill parameter }=\frac{1-\text { force error }}{\text { force error } \cdot\left(\log (\text { duration })^{1.627}\right)}
$$

\section{Statistical Analysis}

Statistical analysis was conducted using $\mathrm{R}$ version 3.4 .2 with statistical significance set at $p<0.05$. The mean and standard deviation of measures taken at baseline were calculated for each group. One-way ANOVAs were utilized to compare the demographics, fitness, anxiety and depression symptomology, and cognitive battery scores of each group of participants at baseline. One-way ANOVA showed no group difference for skill at block one $\left(F_{2,38}=0.03 ; p=0.97\right)$. A general linear mixed model with a random intercept fitted for subjects was utilized using the lme 4 package to take into account the repeated measures design of the study. The model initially included the interaction effect between groups (exercise and a-tDCS, exercise and sham tDCS, and non-exercise and sham tDCS) and time [blocks 14, blocks 5-8, blocks 9-12, and retention (blocks 13 and 14)]. Non-significant interaction terms were dropped from the final model for ease of interpretation. Visual inspection of the QQplots generated for the models showed no significant deviation from normality. A type II Wald $F$ test with the car package was utilized to obtain $p$-values for each model. Effect sizes are 
presented as the beta estimate from the general linear models for the relevant measures.

\section{RESULTS}

Due to a technical impedance error during the application of the a-tDCS, one participant from the exercise and active a-tDCS group was removed from the analysis. Further, one participant from the exercise and active a-tDCS group, and one from the non-exercise and sham tDCS were removed from the analysis after visual inspection of the data indicated a recording error with the motor-learning task. Forty-two participants (age $=44.6 \pm 6.3$, female $=32$, and left handed $=3$ ) had data available for analysis. There were no group differences at baseline in demographic characteristics, mood, fitness levels, or cognitive performance (Table 1). On average, participants had 17 years of education, and were university graduates. There was an average of 6 days between the final learning and retention session. At the completion of the retention session, participants were informed that some participants received sham-tDCS and were asked which form of tDCS they believed they received. Fifty-two percent of participants correctly reported which tDCS protocol was administered, which is similar to the chance-level of blinding reported in tDCS studies involving protocols of greater than $1 \mathrm{~mA}$ (Turi et al., 2019). Across the intervention sessions, the mean percent of age-predicted maximum heart rate (Tanaka et al., 2001) achieved by the end of the first epoch and the end of the fourth epoch were $85 \pm 8 \mathrm{bpm}$ and $90 \pm 7$ for the exercise and active a-tDCS group and $84 \pm 5$ and $90 \pm 6$ for the exercise and sham tDCS group.

\section{Online Change}

Online change refers to the change in motor-skill performance within a session, across blocks. This was assessed by comparing the average skill level of each block throughout the intervention sessions. As displayed in Figure 4, all three groups significantly improved their motor-skill level during the intervention period, demonstrated by a main effect of time $\left(F_{11,444.08}=20.49\right.$; $p=<0.001)$, with skill increasing 15.55 (11.75-19.34 95\% CI) between block 1 and block 12. However, there were no significant differences in motor-skill change between the groups across the intervention sessions, with no main effect of group $\left(F_{2,39.01}=0.02 ; p=0.98\right)$, and no group by time interaction $\left(F_{22,422.08}=1.18 ; p=0.26\right)$. Online change has been visually represented in Figure 5 as the sum of differences between the first and last training blocks of each intervention session $[($ Day1Block4 - Day1Block1 $)+($ Day2Block4 - Day2Block1 $)+$ (Day3Block4 - Day3Block1)].

\section{Offline Change}

Offline change refers to the difference in performance between training sessions. In this study, the offline change was assessed by comparing the average motor-skill level of the final block of each intervention session, with the first block of the following intervention session in separate models (Robertson et al., 2004; Reis et al., 2009). There was a trend toward a decrement in offline change observed between the final block of day one, and the first block of day two, with skill decreasing by -1.98 ( -4.48 to $0.0595 \% \mathrm{CI}$ ), however, the main effect of time was not significant $\left(F_{1,40.22}=38 ; p=0.09\right)$, and did not differ between groups $\left(F_{2,39.01}=1.35 ; p=0.27\right)$. There was no significant group by time interaction observed when assessing offline change between the final block of day one, and the first block of day two $\left(F_{2,38.25}=0.38 ; p=0.69\right)$. Similarly, there was no main time effect observed for the offline change between the final block of day two and the first block of day three $\left(F_{1,41}=0.62 ; p=0.44\right)$, and results did not differ significantly by group $\left(F_{2,39}=0.19 ; p=0.83\right)$. There was also no group by time interaction effect $\left(F_{2,39}=0.38\right.$; $p=0.68$ ). The results did not differ when an average of the final two blocks of an intervention session with the average of the first two blocks of the following intervention session. Offline change has been visually represented in Figure $\mathbf{5}$ as the sum of differences between the first training block of the second intervention session and the last training block of the first intervention session, and the first training block of the third intervention session and the last training block of the second intervention sessions [(Day2Block1 Day1Block4) + (Day3Block1 - Day2Block4)].

\section{Retention of Motor-Skill}

Retention of motor-skill was assessed by comparing the average skill of the four blocks from the final intervention session to the average skill of both blocks completed during the retention session. There was no main effect of time $\left(F_{1,39.23}=2.83 ; p=0.10\right)$ or group $\left(F_{2,38.99}=0.07 ; p=0.93\right)$. There was no significant group by time interaction observed $\left(F_{2,37.36}=0.23 ; p=0.79\right)$. Total learning has been visually represented in Figure 5 to include the retention portion of the task. Total learning is represented as the sum of online change, offline change, and the difference between the first block of retention and the last block of learning (RetentionBlock1 - Day3Block4), and the difference between the first and last block of the retention session (RetentionBlock2 RetentionBlock1).

Sensitivity analysis revealed that adding covariates of age, sex, fitness (relative $\mathrm{VO}_{2}$ peak), manual dexterity, cognitive function, the number of days between the intervention and retention session, and anxiety did not change the results of the study (all p's > 0.05).

\section{DISCUSSION}

This study investigated the effects of a short-term high-intensity interval exercise intervention with and without a-tDCS applied over M1 on motor-skill change across three sessions, and retention 5-7 days later, in middle-aged adults. Although motorskill learning occurred, there was no evidence of increased learning or retention when exercise was performed before the motor task, or when exercise was combined with a-tDCS. The current study is the first to our knowledge which has utilized high-intensity interval exercise and a-tDCS techniques within the one session targeting motor-skill learning, and one of the few investigations of motor learning in middleaged adults. 
TABLE 1 | Group demographic, motor dexterity, self-reported mood, fitness, and cognitive performance characteristics and differences determined by one-way ANOVAs.

\begin{tabular}{|c|c|c|c|c|}
\hline & $\begin{array}{c}\text { Exercise and } \\
\text { sham } \text { tDCS } n=15 \\
\text { Mean } \pm \text { SD }\end{array}$ & $\begin{array}{c}\text { Exercise and active } \\
\text { a-tDCS } n=13 \\
\text { Mean } \pm \text { SD }\end{array}$ & $\begin{array}{c}\text { Non-exercise and } \\
\text { sham tDCS } n=14 \\
\text { Mean } \pm \text { SD }\end{array}$ & $p$ \\
\hline Age (years) & $45 \pm 5$ & $43 \pm 8$ & $45 \pm 6$ & $\begin{array}{c}F_{2,39}=0.4 \\
p=0.70\end{array}$ \\
\hline Height (cm) & $172 \pm 10$ & $175 \pm 10$ & $172 \pm 9$ & $\begin{array}{c}F_{2,39}=0.3 \\
p=0.72\end{array}$ \\
\hline Weight (kg) & $73 \pm 14$ & $77 \pm 11$ & $72 \pm 14$ & $\begin{array}{c}F_{2,39}=0.5 \\
p=0.52\end{array}$ \\
\hline Female $n(\%)$ & $11(73)$ & $11(77)$ & $10(71)$ & - \\
\hline Highest qualification* & $6 \pm 2$ & $5 \pm 2$ & $6 \pm 1$ & $\begin{array}{c}F_{2,39}=0.3 \\
p=0.77\end{array}$ \\
\hline Education (years) & $17 \pm 3$ & $17 \pm 3$ & $17 \pm 4$ & $\begin{array}{c}F_{2,39}=0.004 \\
p=0.99\end{array}$ \\
\hline Purdue pegboard sum of scores (no. of pegs) & $56 \pm 5$ & $54 \pm 6$ & $57 \pm 7$ & $\begin{array}{c}F_{2,39}=0.8 \\
p=0.46\end{array}$ \\
\hline Goldberg anxiety score & $0.5 \pm 1.1$ & $0.5 \pm 0.7$ & $0.6 \pm 1.7$ & $\begin{array}{c}F_{2,39}=0.03 \\
p=0.97\end{array}$ \\
\hline Goldenberg depression score & $0.6 \pm 1.8$ & $0.6 \pm 0.6$ & $0.1 \pm 0.3$ & $\begin{array}{c}F_{2,39}=0.9 \\
p=0.42\end{array}$ \\
\hline MVPA (min week ${ }^{-1}$ ) & $660 \pm 1002$ & $483 \pm 506$ & $407 \pm 471$ & $\begin{array}{c}F_{2,33}=0.4 \\
p=0.67\end{array}$ \\
\hline$\dot{\mathrm{V}} \mathrm{O}_{2}$ peak $\left(\mathrm{mL} \mathrm{min}^{-1} \mathrm{~kg}^{-1}\right)$ & $40 \pm 8$ & $37 \pm 11$ & $40 \pm 7$ & $\begin{array}{c}F_{2,39}=0.7 \\
p=0.51\end{array}$ \\
\hline Stroop accuracy & $100 \pm 1$ & $99 \pm 3$ & $100 \pm 2$ & $\begin{array}{c}F_{2,38}=2.6 \\
p=0.09\end{array}$ \\
\hline Stroop reaction time (ms) & $752 \pm 65$ & $809 \pm 132$ & $741 \pm 109$ & $\begin{array}{c}F_{2,38}=1.6 \\
p=0.22\end{array}$ \\
\hline Wisconsin card sorting task correct (\%) & $73 \pm 15$ & $78 \pm 10$ & $75 \pm 18$ & $\begin{array}{c}F_{2,39}=0.5 \\
p=0.63\end{array}$ \\
\hline Wisconsin card sorting task reaction time (ms) & $1901 \pm 540$ & $1747 \pm 276$ & $1814 \pm 732$ & $\begin{array}{c}F_{2,39}=0.3 \\
p=0.76\end{array}$ \\
\hline Go/No-Go task trial 1 accuracy (\%) & $96 \pm 2$ & $96 \pm 3$ & $94 \pm 3$ & $\begin{array}{c}F_{2,39}=1.6 \\
p=0.21\end{array}$ \\
\hline Go/No-Go task reaction time (ms) & $476 \pm 46$ & $477 \pm 32$ & $480 \pm 43$ & $\begin{array}{c}F_{2,39}=0.05 \\
p=0.96\end{array}$ \\
\hline Go/No-Go task trial 2 accuracy (\%) & $94 \pm 6$ & $91 \pm 9$ & $88 \pm 6$ & $\begin{array}{c}F_{2,39}=2.1 \\
p=0.13\end{array}$ \\
\hline Go/No-Go task reaction time (ms) & $493 \pm 118$ & $530 \pm 79$ & $536 \pm 81$ & $\begin{array}{c}F_{2,39}=0.9 \\
p=0.42\end{array}$ \\
\hline
\end{tabular}

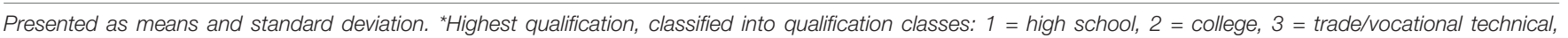
4 = diploma, 5 = bachelors, 6 = honors $/$ graduate diploma, 7 = masters, $8=$ doctorate.

The non-significant effects of high-intensity interval exercise and a-tDCS on motor learning in the current study should be discussed in the context of the study population. Older adults $(60$ years+) may have an impaired offline change when compared to young adults (Ehsani et al., 2015). However, middle-aged adults performed more similarly to younger than older adults on a perceptual motor-skill acquisition task (Kennedy and Raz, 2005). As the current middle-aged cohort was a highly educated, healthy population, it is unlikely that cognitive decline had occurred to a stage of offline change impairment. Previous literature has focused primarily on young adults (Reis et al., 2009; Mang et al., 2014; Stavrinos and Coxon, 2017), and although the current study shared several similarities with the intensity and timing of the high-intensity interval exercise (Mang et al., 2014; Stavrinos and Coxon, 2017) and a-tDCS protocols (Reis et al., 2009; WatersMetenier et al., 2014) in relation to the motor task, interventions may need to be tailored to specific age and sociodemographic populations in future research.

High-intensity interval exercise did not improve the retention of a motor-skill 5-7 days after the intervention. This contrasts 


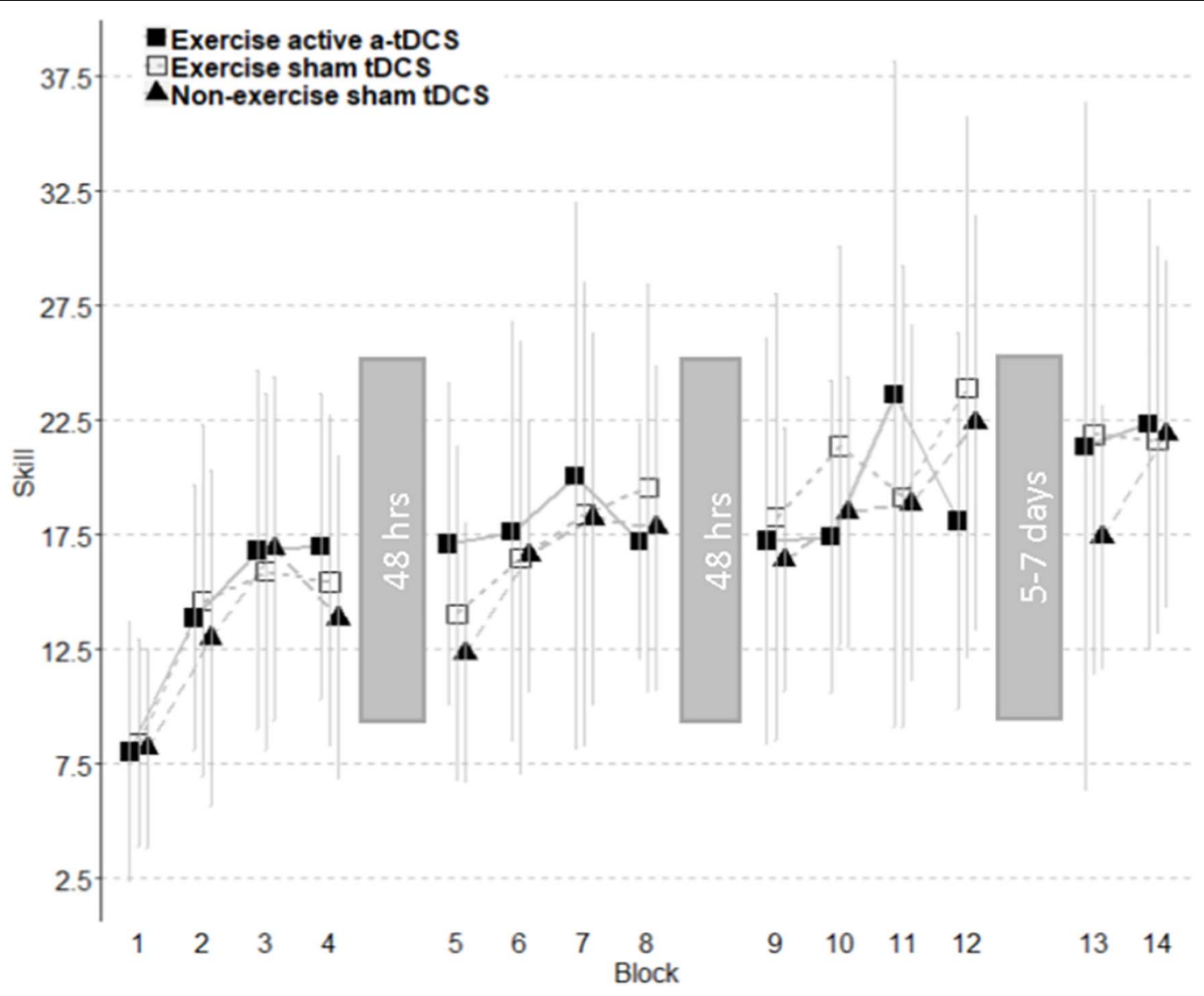

FIGURE 4 | Improvement of motor-skill across all blocks. Skill change curves are separated into individual sessions with the time between sessions displayed within grayed blocks. The analysis of subcomponents showed an increase in motor skill over three intervention sessions observed across all groups. A trend toward a decrement of skill was observed between the first and second intervention session. Retention did not differ between groups. Error bars represent standard deviations.

previous literature which has shown enhanced retention of motor-skill following high-intensity interval exercise (Roig et al., 2012; Skriver et al., 2014). The lack of benefit observed for a-tDCS on the retention of a motor-skill is in agreement with others who have similarly found no change in the long-term retention of a motor skill following a similar brain stimulation protocol, tested between eight and 30 days post learning (Reis et al., 2009). Previously, studies which have observed increased retention with high-intensity interval exercise (Roig et al., 2012; Skriver et al., 2014) have implemented multiple retention sessions. The repetitive testing at 1 - and 24 -h post-exercise may have provided additional consolidation opportunities, muddying the effects of exercise on retention. The consolidation of skilled finger movements is highly susceptible to interference, particularly within the first $6 \mathrm{~h}$ after learning a task (Krakauer and Shadmehr, 2006). This may have been exacerbated in the exercise group, as cognitive functions benefit from acute exercise for up to 2 -h post-exercise (Basso et al., 2015). Thus, the exercise group may have received additional positive benefits from the exercise, when a 1-h consolidation was used. Further, it has previously been postulated that a 24 -h retention session may have impacted the results seen at 48-h through additional consolidation (Korman et al., 2003). Future research should implement designs in which consolidation and retention are measured as separate entities to confirm the effects of exercise on the consolidation and retention of motor-skill learning. In the current study, no enhancement in a single retention session conducted 5-7 days after the motor-skill intervention was observed.

Online change was assessed by comparing the average skill level of each block throughout the learning phase. A positive online change was observed, however, there were no significant differences between groups. This is in line with previous literature, which has suggested that both a-tDCS during (Reis et al., 2009), and high-intensity interval exercise prior (Mang et al., 2014; Stavrinos and Coxon, 2017), to a task individually enhance learning through enhanced offline change, and not online change, of motor-skill learning in young adults.

Contrary to the hypothesis, the current study found no benefit of high-intensity interval exercise, or high-intensity 


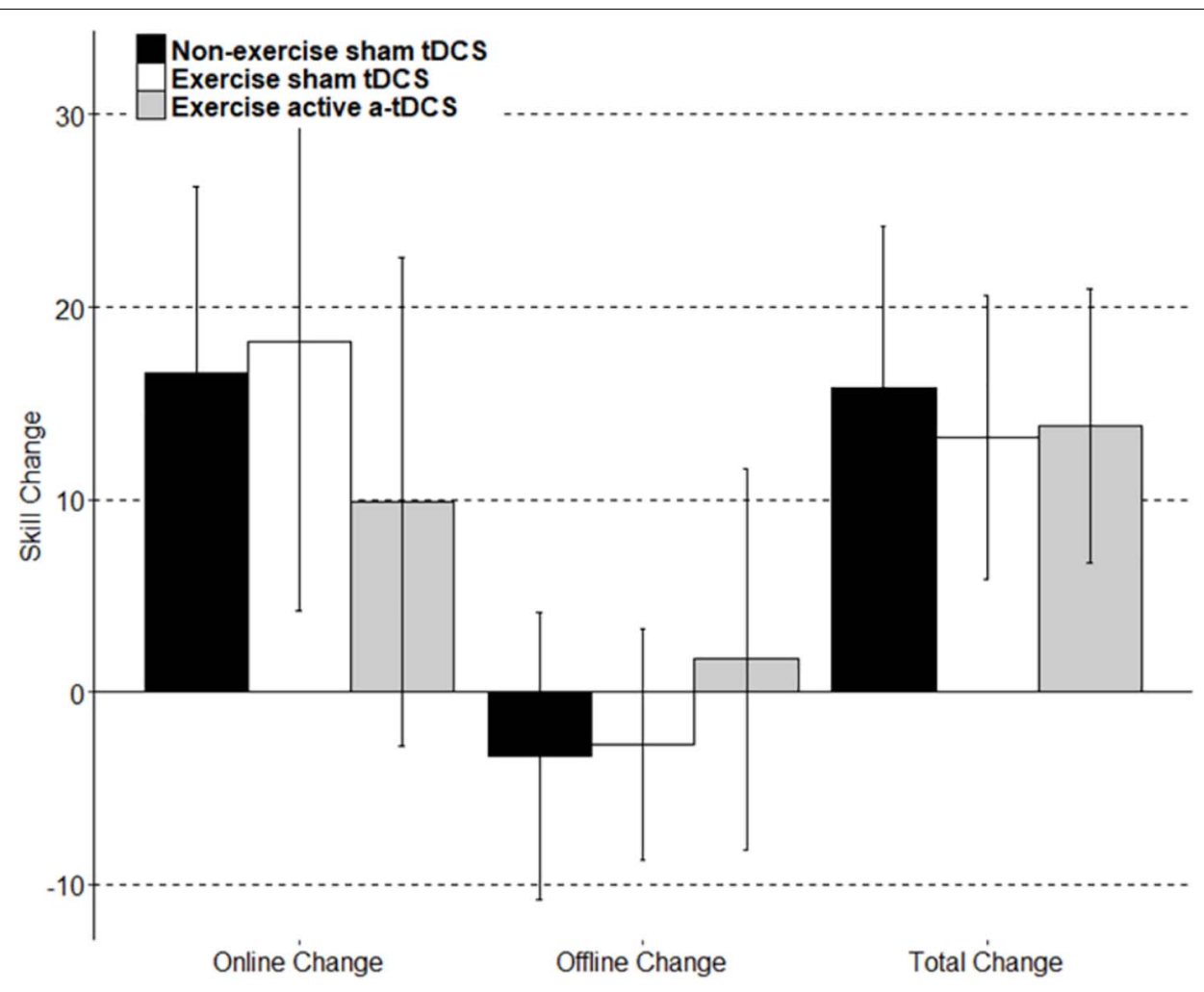

FIGURE 5 | A visual representation of the group mean skill change score of subcomponents. Online change: the sum of difference of scores between only the last block and first block of each intervention session. Offline change: the sum of scores from the differences between only the first block of day two and the last block of day 1 , and the first block of day three and the last block of day two. Total change: the sum of online change, offline change, the difference between the first block of retention and the last block of day 3 , and the difference between the final retention block and the first retention block.

interval exercise and a-tDCS to the offline phase of a motorskill. Previously, exercise has improved the offline phase of a motor-skill in young adults after a single session of exercise (Mang et al., 2014; Stavrinos and Coxon, 2017). Previous studies which employed similar exercise protocols, tested the offline change of a motor-skill 5-h (Stavrinos and Coxon, 2017) and 24-h (Mang et al., 2014) after a single exposure to a motor-learning task, or a repeat exposure at a $24-\mathrm{h}$ retention was utilized (Roig et al., 2012), whereas the current study assessed offline change between intervention sessions, which were 48 -h apart. Enhancement in offline change by highintensity exercise may be transient and therefore, may not have been observed in the current study due to the protracted time between sessions. Offline change may be influenced by the inclusion, or exclusion, of refamiliarization to the task, which may overcome the "warm-up decrement" sometimes observed in motor-learning literature (Adams, 1961). As there were no group differences between groups in the current study, it is unlikely that the exclusion of re-familiarization task impacted the outcomes. It should also be considered that in the current investigation comparisons were made to the first learning block. Future research may benefit from the inclusion of a baseline block prior to intervention. However, this can be difficult to balance as baseline testing may influence the observed learning curve.
The timing of the application of a-tDCS may have also contributed to the results of the current study. The current study applied a-tDCS during the motor task, which has previously enhanced the offline phase of motor-skill learning (Reis et al., 2009; Waters-Metenier et al., 2014). Exercise performed prior to a-tDCS has been hypothesized to provide an environment conducive to increased neuroplasticity (Mellow et al., 2020) due to an increase in the neuromodulator BDNF following exercise (Saucedo Marquez et al., 2015; Steinberg et al., 2019). Recent research, however, (Baltar et al., 2018) has demonstrated that a-tDCS applied prior to high-intensity exercise resulted in an inhibitory effect of cortical excitability for a 20-min period, suggesting that homeostatic plasticity may moderate the interaction between the interventions. Baltar et al. (2018) suggested that the high-intensity running protocol utilized may have caused fatigue, and therefore decreased cortical excitability, compared to the moderate-intensity running protocol, which caused excitatory effects. Recent evidence has also observed increases in motor-skill acquisition after moderate-intensity exercise (Statton et al., 2015).Cycling has been associated with improved motor and cognitive performance (Lambourne and Tomporowski, 2010) completed both during after exercise, including those designed to induce fatigue, and cognitive performance after exercise (Lambourne and Tomporowski, 2010). However, it may be that when combined with tDCS, 
in the current age population the high-intensity cycling may have induced a non-beneficial level of fatigue was induced. Perhaps in future works a lower exercise-intensity may be more beneficial. As this study did not measure motor evoked potentials, it cannot be confirmed whether the application of high-intensity exercise prior to a-tDCS had unexpected inhibitory effects. As there were no significant group effects, this was unlikely the leading cause for the non-significant results in the current study. Moderate-intensity exercise may be appropriate, as it has shown potential as a mechanism to enhance motor performance in older adults, proposed to be achieved through higher beta activity following the exercise (Hübner et al., 2018). Although the current investigation utilized a tDCS montage which has successfully enhanced motor sequence learning in a previous investigation (Waters-Metenier et al., 2014), modeling was not utilized to confirm the accuracy and specificity of the montage. tDCS montage modeling in future investigations is recommended to increase the specificity of tDCS application. One additional possibility is that following exercise, sweat on the scalp may have influenced the flow of current produced by tDCS (Horvath et al., 2014), by shunting the current away from the target area of the scalp and reducing the amount of stimulation to M1. While this cannot be ruled out, given benefit of tDCS applied during or after exercise on other cognitive functions (Ward et al., 2017), this is less likely to be the cause of the current findings. Although, the differences in application of tDCS between the current investigation should be considered. The use of a neoprene headcap and saline soaked electrodes, may have made the current set up more responsive to sweating compared to the discussed investigation (Ward et al., 2017) which utilized smaller, gel electrodes fixed by elastic band.

Although the non-significant effects of a-tDCS and highintensity interval exercise on motor learning were unexpected in the current study, it is notable that two recent studies investigating the combination of a-tDCS and aerobic exercise on executive functions also reported no significant benefit on cognitive performance (Hendy et al., 2019; Thomas et al., 2020). While these studies had differences in protocol, including difference in cognitive tasks and stimulated regions, making them not directly comparable, these non-significant findings also reflect the ongoing difficulty in replicability of results using tDCS (Horvath et al., 2014), and to a more limited extent, aerobic exercise (Mellow et al., 2020). Individual differences in the response to tDCS have been observed (Filmer et al., 2019), and it may be that exercise induces individualized responses too (Hendy et al., 2019). These differences have been shown to include response modulation from base levels of gamma-aminobutyric acid and glutamate, known to relate to neurochemical excitability (Filmer et al., 2019), and the direction of response achieved (excitatory or inhibitory) (Wiethoff et al., 2014). However, as this investigation did not include a t-DCS only group, the effects of exercise on individual responses to tDCS cannot be determined. Further work is needed to establish the determinants of individual responses to both tDCS and aerobic exercise, both alone and in combination, before effective protocols can be potentially developed.
Although this study contained well-balanced groups, the samples high level of education, health, and larger proportion of female participants may limit the applicability of the findings to wider populations, as women may experience altered cortical excitability following stimulation dependent on the stage of their menstrual cycle (Inghilleri et al., 2004), and potentially lower excitation compared to men (Russell et al., 2017). However, the non-significant effect of sex within the models suggests this did not affect the outcome of the current study. Future research should extend to more comprehensive representations of the middle-aged adult population. Future research should also investigate the optimization of the timing between intervention sessions, and the timing at which exercise and a-tDCS are implemented within a session, as timing may be important in overcoming homeostatic plasticity. The addition of neurophysiology measures to investigate changes to cortical excitability and inhibition following these interventions, will further enhance future research in determining the interaction between exercise and non-invasive brain stimulation protocols. Additionally, investigations into the type of exercise, and the dose of a-tDCS will provide more insight into whether there is a beneficial combination for enhanced motor-skill learning in this population.

\section{CONCLUSION}

The development and maintenance of motor-skills are a vital component of healthy aging. This study provides the first investigation of the effects of combining a short-term highintensity interval exercise intervention with a-tDCS on a novel motor task in middle-aged adults. The current study adds to the limited literature regarding motor-skill learning in a middle-aged population, providing a methodological framework for future studies. Although the current study was not able to confirm the prescription of exercise required to enhance motor-skill learning in middle-aged adults, it demonstrated that short-term intervention consisting of three 20-min high-intensity interval exercise sessions did not increase motor-skill learning or retention 5-7 days later, either individually, or when paired with a-tDCS applied during a motor-learning task compared to a control group.

\section{DATA AVAILABILITY STATEMENT}

The raw data supporting the conclusions of this article will be made available by the corresponding author, without undue reservations.

\section{ETHICS STATEMENT}

The studies involving human participants were reviewed and approved by the University of Canberra and the Australian National University Human Research Ethics Committee (HREC 16-121, and 2016/322, respectively). The 
patients/participants provided their written informed consent to participate in this study.

\section{AUTHOR CONTRIBUTIONS}

CQ: conceptualisation, methodology, investigation, formal analysis, data curation, and writing - orgininal draft preparation. BR, DP, and JN: conceptualisation, methodology, formal analysis, and writing - orgininal draft preparation. JC: conceptualisation, methodology, formal analysis, data curation, writing - review and editing, and software. NC: conceptualisation, methodology, and writing - review and editing. SA: conceptualisation, methodology, investigation, formal analysis, and writing - review and editing.

\section{REFERENCES}

Adams, J. A. (1961). The second facet of forgetting: a review of warm-up decrement. Psychol. Bull. 58:257. doi: 10.1037/h0044798

Andrews, S. C., Curtin, D., Hawi, Z., Wongtrakun, J., Stout, J. C., and Coxon, J. P. (2020). Intensity matters: high-intensity interval exercise enhances motor cortex plasticity more than moderate exercise. Cereb. Cortex 30, 101-112. doi: 10.1093/cercor/bhz075

Antal, A., Alekseichuk, I., Bikson, M., Brockmöller, J., Brunoni, A. R., Chen, R., et al. (2017). Low intensity transcranial electric stimulation: safety, ethical, legal regulatory and application guidelines. Clin. Neurophysiol. 128, 1774-1809. doi: 10.1016/j.clinph.2017.06.001

Baltar, A., Nogueira, F., Marques, D., Carneiro, M., and Monte-Silva, K. (2018). Evidence of the homeostatic regulation with the combination of transcranial direct current stimulation and physical activity. Am. J. Phys. Med. Rehabil. 97, 727-733. doi: 10.1097/phm.0000000000000956

Basso, J. C., Shang, A., Elman, M., Karmouta, R., and Suzuki, W. A. (2015). Acute exercise improves prefrontal cortex but not hippocampal function in healthy adults. J. Int. Neuropsychol. Soc. 21, 791-801. doi: 10.1017/s13556177150 $0106 \mathrm{x}$

Bestmann, S., de Berker, A. O., and Bonaiuto, J. (2015). Understanding the behavioural consequences of noninvasive brain stimulation. Trends Cogn. Sci. 19, 13-20. doi: 10.1016/j.tics.2014.10.003

Buchheit, M., and Laursen, P. B. (2013). High-intensity interval training, solutions to the programming puzzle. Sports Med. 43, 313-338. doi: 10.1007/s40279-0130029-x

Caligiuri, M. P., and Ellwanger, J. (2000). Motor and cognitive aspects of motor retardation in depression. J. Affect. Disord. 57, 83-93. doi: 10.1016/s01650327(99)00068-3

Cunha, C., Brambilla, R., and Thomas, K. L. (2010). A simple role for BDNF in learning and memory? Front. Mol. Neurosci. 3:1. doi: 10.3389/neuro.02.001. 2010

DaSilva, A. F., Volz, M. S., Bikson, M., and Fregni, F. (2011). Electrode positioning and montage in transcranial direct current stimulation. J. Vis. Exp. 51:2744.

Ehsani, F., Abdollahi, I., Bandpei, M. A. M., Zahiri, N., and Jaberzadeh, S. (2015). Motor learning and movement performance: older versus younger adults. Basic Clin. Neurosci. 6:231.

Filmer, H. L., Ehrhardt, S. E., Bollmann, S., Mattingley, J. B., and Dux, P. E. (2019). Accounting for individual differences in the response to tDCS with baseline levels of neurochemical excitability. Cortex 115, 324-334. doi: 10.1016/j.cortex. 2019.02.012

Fox, C. J., Mueller, S. T., Gray, H. M., Raber, J., and Piper, B. J. (2013). Evaluation of a short-form of the berg card sorting test. PLoS One 8:e63885. doi: 10.1371/ journal.pone.0063885

Fritsch, B., Reis, J., Martinowich, K., Schambra, H. M., Ji, Y., Cohen, L. G., et al. (2010). Direct current stimulation promotes BDNF-dependent synaptic plasticity: potential implications for motor learning. Neuron 66, 198-204. doi: 10.1016/j.neuron.2010.03.035
All authors contributed to the article and approved the submitted version.

\section{FUNDING}

SA was supported by a fellowship from the Huntington's Disease Society of America, and also received support from the Australian National Health and Medical Research Council Centre for Research Excellence in Cognitive Health.

\section{ACKNOWLEDGMENTS}

The authors thank the participants.

Goldberg, D., Bridges, K., Duncan-Jones, P., and Grayson, D. (1988). Detecting anxiety and depression in general medical settings. Br. Med. J. 297, 897-899. doi: $10.1136 /$ bmj.297.6653.897

Hendy, A. M., Macpherson, H., Nuzum, N. D., Della Gatta, P. A., Alexander, S. E., Hoy, K. E., et al. (2019). High intensity aerobic exercise does not prime the brain for anodal transcranial direct current stimulation. Brain Stimul. 12, 1086-1088. doi: 10.1016/j.brs.2019.04.007

Horvath, J. C., Carter, O., and Forte, J. D. (2014). Transcranial direct current stimulation: five important issues we aren't discussing (but probably should be). Front. Syst. Neurosci. 8:2. doi: 10.3389/fnsys.2014.00002

Hübner, L., Godde, B., and Voelcker-Rehage, C. (2018). Acute exercise as an intervention to trigger motor performance and EEG beta activity in older adults. Neural Plast. 2018:4756785.

Inghilleri, M., Conte, A., Curra, A., Frasca, V., Lorenzano, C., and Berardelli, A. (2004). Ovarian hormones and cortical excitability. An rTMS study in humans. Clin. Neurophysiol. 115, 1063-1068. doi: 10.1016/j.clinph.2003.12.003

Kennedy, K. M., and Raz, N. (2005). Age, sex and regional brain volumes predict perceptual-motor skill acquisition. Cortex 41, 560-569. doi: 10.1016/s00109452(08)70196-5

Kober, S. E., Reichert, J. L., Neuper, C., and Wood, G. (2016). Interactive effects of age and gender on EEG power and coherence during a short-term memory task in middle-aged adults. Neurobiol. Aging 40, 127-137. doi: 10.1016/j. neurobiolaging.2016.01.015

Korman, M., Raz, N., Flash, T., and Karni, A. (2003). Multiple shifts in the representation of a motor sequence during the acquisition of skilled performance. Proc. Natl. Acad. Sci. U.S.A. 100, 12492-12497. doi: 10.1073/pnas. 2035019100

Kowalski-Trakofler, K. M., Steiner, L. J., and Schwerha, D. J. (2005). Safety considerations for the aging workforce. Saf. Sci. 43, 779-793. doi: 10.1016/j. ssci.2005.08.014

Krakauer, J. W., and Shadmehr, R. (2006). Consolidation of motor memory. Trends Neurosci. 29, 58-64.

Kronberg, G., Bridi, M., Abel, T., Bikson, M., and Parra, L. C. (2017). Direct current stimulation modulates LTP and LTD: activity dependence and dendritic effects. Brain Stimul. 10, 51-58. doi: 10.1016/j.brs.2016.10.001

Lafayette Instrument Company (2015). Purdue Pegboard Test-User Instructions. Lafayette, IN: Lafayette Instrument Company.

Lambourne, K., and Tomporowski, P. (2010). The effect of exercise-induced arousal on cognitive task performance: a meta-regression analysis. Brain Res. 1341, 12-24. doi: 10.1016/j.brainres.2010.03.091

Langenecker, S. A., Zubieta, J.-K., Young, E. A., Akil, H., and Nielson, K. A. (2007). A task to manipulate attentional load, set-shifting, and inhibitory control: convergent validity and test-retest reliability of the Parametric Go/NoGo Test. J. Clin. Exp. Neuropsychol. 29, 842-853. doi: 10.1080/138033906011 47611

Li, L., Uehara, K., and Hanakawa, T. (2015). The contribution of interindividual factors to variability of response in transcranial direct current stimulation studies. Front. Cell. Neurosci. 9:181. doi: 10.3389/fncel.2015.00181 
Loprinzi, P. D., Harris, F., McRaney, K., Chism, M., Deming, R., Jones, T., et al. (2019). Effects of acute exercise and learning strategy implementation on memory function. Medicina 55:568. doi: 10.3390/medicina55090568

Macpherson, H., Teo, W.-P., Schneider, L. A., and Smith, A. E. (2017). A lifelong approach to physical activity for brain health. Front. Aging Neurosci. 9:147. doi: 10.3389/fnagi.2017.00147

Mandolesi, L., Polverino, A., Montuori, S., Foti, F., Ferraioli, G., Sorrentino, P., et al. (2018). Effects of physical exercise on cognitive functioning and wellbeing: biological and psychological benefits. Front. Psychol. 9:509. doi: 10.3389/fpsyg. 2018.00509

Mang, C. S., Snow, N. J., Campbell, K. L., Ross, C. J., and Boyd, L. A. (2014). A single bout of high-intensity aerobic exercise facilitates response to paired associative stimulation and promotes sequence-specific implicit motor learning. Am. J. Physiol. Heart Circ. Physiol. 117, 1325-1336. doi: 10.1152/japplphysiol. 00498.2014

Mellow, M. L., Goldsworthy, M. R., Coussens, S., and Smith, A. E. (2020). Acute aerobic exercise and neuroplasticity of the motor cortex: a systematic review. J. Sci. Med. Sport 23, 408-414. doi: 10.1016/j.jsams.2019.10.015

Mooney, R. A., Cirillo, J., and Byblow, W. D. (2019). Neurophysiological mechanisms underlying motor skill learning in young and older adults. Exp. Brain Res. 237, 2331-2344. doi: 10.1007/s00221-019-05599-8

Nitsche, M. A., Fricke, K., Henschke, U., Schlitterlau, A., Liebetanz, D., Lang, N., et al. (2003a). Pharmacological modulation of cortical excitability shifts induced by transcranial direct current stimulation in humans. J. App. Physiol. 553, 293-301. doi: 10.1113/jphysiol.2003.049916

Nitsche, M. A., Schauenburg, A., Lang, N., Liebetanz, D., Exner, C., Paulus, W., et al. (2003b). Facilitation of implicit motor learning by weak transcranial direct current stimulation of the primary motor cortex in the human. J. Cogn. Neurosci. 15, 619-626. doi: 10.1162/089892903321662994

Pozzo-Miller, L. D., Gottschalk, W., Zhang, L., McDermott, K., Du, J., Gopalakrishnan, R., et al. (1999). Impairments in high-frequency transmission, synaptic vesicle docking, and synaptic protein distribution in the hippocampus of BDNF knockout mice. J. Neurosci. 19, 4972-4983. doi: 10.1523/jneurosci.1912-04972.1999

Reis, J., Schambra, H. M., Cohen, L. G., Buch, E. R., Fritsch, B., Zarahn, E., et al. (2009). Noninvasive cortical stimulation enhances motor skill acquisition over multiple days through an effect on consolidation. Proc. Natl. Acad. Sci. U.S.A. 106, 1590-1595. doi: 10.1073/pnas.0805413106

Ren, J., Wu, Y. D., Chan, J. S., and Yan, J. H. (2013). Cognitive aging affects motor performance and learning. Geriatr. Gerontol. Int. 13, 19-27. doi: 10.1111/j. 1447-0594.2012.00914.x

Robertson, E. M., Pascual-Leone, A., and Miall, R. C. (2004). Current concepts in procedural consolidation. Nat. Rev. Neurosci. 5, 576-582. doi: 10.1038/nrn1426

Roig, M., Skriver, K., Lundbye-Jensen, J., Kiens, B., and Nielsen, J. B. (2012). A single bout of exercise improves motor memory. PLoS One 7:e44594. doi: 10.1371/journal.pone.0044594

Russell, M. J., Goodman, T. A., Visse, J. M., Beckett, L., Saito, N., Lyeth, B. G., et al. (2017). Sex and electrode configuration in transcranial electrical stimulation. Front. Psychiatry 8:147. doi: 10.3389/fpsyt.2017.00147

Saucedo Marquez, C. M., Vanaudenaerde, B., Troosters, T., and Wenderoth, N. (2015). High-intensity interval training evokes larger serum BDNF levels compared with intense continuous exercise. J. Appl. Physiol. 119, 1363-1373. doi: 10.1152/japplphysiol.00126.2015

Schmolesky, M. T., Webb, D. L., and Hansen, R. A. (2013). The effects of aerobic exercise intensity and duration on levels of brain-derived neurotrophic factor in healthy men. J. Sports Sci. Med. 12:502.

Schoenfeld, D. (2015). Statistical Considerations for Clinical Trials and Scientific Experiments. Available online at: http://hedwig.mgh.harvard.edu/sample_size/ size.html\#ssize (accessed March 2019).

Seidler, R. D., Bernard, J. A., Burutolu, T. B., Fling, B. W., Gordon, M. T., Gwin, J. T., et al. (2010). Motor control and aging: links to age-related brain structural, functional, and biochemical effects. Neurosci. Biobehav. Rev. 34, 721-733. doi: 10.1016/j.neubiorev.2009.10.005

Skriver, K., Roig, M., Lundbye-Jensen, J., Pingel, J., Helge, J. W., Kiens, B., et al. (2014). Acute exercise improves motor memory: exploring potential biomarkers. Neurobiol. Learn. Mem. 116, 46-58. doi: 10.1016/j.nlm.2014.08. 004
Stagg, C., Jayaram, G., Pastor, D., Kincses, Z., Matthews, P., and Johansen-Berg, H. (2011). Polarity and timing-dependent effects of transcranial direct current stimulation in explicit motor learning. Neuropsychologia 49, 800-804. doi: 10.1016/j.neuropsychologia.2011.02.009

Statton, M. A., Encarnacion, M., Celnik, P., and Bastian, A. J. (2015). A single bout of moderate aerobic exercise improves motor skill acquisition. PLoS One 10:e0141393. doi: 10.1371/journal.pone.0141393

Stavrinos, E. L., and Coxon, J. P. (2017). High-intensity interval exercise promotes motor cortex disinhibition and early motor skill consolidation. J. Cogn. Neurosci. 29, 593-604. doi: 10.1162/jocn_a_01078

Steinberg, F., Pixa, N. H., and Fregni, F. (2019). A review of acute aerobic exercise and transcranial direct current stimulation effects on cognitive functions and their potential synergies. Front. Hum. Neurosci. 12:534. doi: 10.3389/fnhum. 2018.00534

Stroop, J. R. (1935). Studies of interference in serial verbal reactions. J. Exp. Psychol. 18:643. doi: $10.1037 / \mathrm{h} 0054651$

Tanaka, H., Monahan, K. D., and Seals, D. R. (2001). Age-predicted maximal heart rate revisited. J. Am. Coll. Cardiol. 37, 153-156. doi: 10.1016/s0735-1097(00) 01054-8

Thomas, F., Pixa, N., Berger, A., Cheng, M.-Y., Doppelmayr, M., and Steinberg, F. (2020). Neither cathodal nor anodal transcranial direct current stimulation on the left dorsolateral prefrontal cortex alone or applied during moderate aerobic exercise modulates executive function. Neuroscience 443, 71-83. doi: 10.1016/j.neuroscience.2020.07.017

Thomas, R., Johnsen, L. K., Geertsen, S. S., Christiansen, L., Ritz, C., Roig, M., et al. (2016). Acute exercise and motor memory consolidation: the role of exercise intensity. PLoS One 11:e0159589. doi: 10.1371/journal.pone.0159589

Turi, Z., Csifcsák, G., Boayue, N. M., Aslaksen, P., Antal, A., Paulus, W., et al. (2019). Blinding is compromised for transcranial direct current stimulation at $1 \mathrm{~mA}$ for $20 \mathrm{~min}$ in young healthy adults. Eur. J. Neurosci. 50, 3261-3268. doi: $10.1111 /$ ejn.14403

Vanhees, L., De Sutter, J., Geladas, N., Doyle, F., Prescott, E., Cornelissen, V., et al. (2012). Importance of characteristics and modalities of physical activity and exercise in defining the benefits to cardiovascular health within the general population: recommendations from the EACPR (Part I). Eur. J. Prev. Cardiol. 19, 670-686. doi: 10.1177/2047487312437059

Wanner, P., Cheng, F.-H., and Steib, S. (2020). Effects of acute cardiovascular exercise on motor memory encoding and consolidation: a systematic review with meta-analysis. Neurosci. Biobehav. Rev. 116, 365-381. doi: 10.1016/j. neubiorev.2020.06.018

Ward, N., Paul, E., Watson, P., Cooke, G., Hillman, C., Cohen, N., et al. (2017). Enhanced learning through multimodal training: evidence from a comprehensive cognitive, physical fitness, and neuroscience intervention. Sci. Rep. 7:5808.

Waters-Metenier, S., Husain, M., Wiestler, T., and Diedrichsen, J. (2014). Bihemispheric transcranial direct current stimulation enhances effectorindependent representations of motor synergy and sequence learning. J. Neurosci. 34, 1037-1050. doi: 10.1523/jneurosci.2282-13.2014

Wiethoff, S., Hamada, M., and Rothwell, J. C. (2014). Variability in response to transcranial direct current stimulation of the motor cortex. Brain Stimul. 7, $468-475$.

Yang, J., Ruchti, E., Petit, J.-M., Jourdain, P., Grenningloh, G., Allaman, I., et al. (2014). Lactate promotes plasticity gene expression by potentiating NMDA signaling in neurons. Proc. Natl. Acad. Sci. U.S.A. 111, 12228-12233. doi: $10.1073 /$ pnas. 1322912111

Conflict of Interest: The authors declare that the research was conducted in the absence of any commercial or financial relationships that could be construed as a potential conflict of interest.

Copyright (C) 2021 Quinlan, Rattray, Pryor, Northey, Coxon, Cherbuin and Andrews. This is an open-access article distributed under the terms of the Creative Commons Attribution License (CC BY). The use, distribution or reproduction in other forums is permitted, provided the original author(s) and the copyright owner(s) are credited and that the original publication in this journal is cited, in accordance with accepted academic practice. No use, distribution or reproduction is permitted which does not comply with these terms. 